\title{
A Study on Numerical Solutions for Rotating Flows of a Third Grade Fluid with Partial Slip Conditions
}

\author{
R.Lakshmi $^{[1]}$, S. Maheswari ${ }^{[2]}$ \\ 1. Assistant Professor, Department of Mathematics, PSGR Krishnammal College for women, Coimbatore, \\ Tamil Nadu, India \\ 2. Assistant Professor, Department of Mathematics, Rathinam College Of Arts \& Science, Coimbatore, \\ Tamil Nadu, India.
}

\begin{abstract}
In this paper rotating flow of a third grade fluid past a porous plate with partial slip is studied The nonlinear boundary value problem is solved using finite difference method. The variations of velocity components for various values of partial parameter $\lambda_{1}$ are discussed the results are reported for conclusion.

Keyword: third grade fluid, partial slip parameter, finite difference method, non - Newtonian fluid.
\end{abstract}

\section{Introduction}

Materials such as polymer solutions or melts, drilling mud, clastomers, certain oils and greases and many other emulsions are classified as non-Newtonian fluids. Due to complexity of fluids, there are many models describing the properties, but not all of non-Newtonian fluids. These models, however, cannot predict all the behaviours of non-Newtonian fluids, for example, normal stress differences, shear thinning or shear thickening, shear relaxation, elastic and memory effects etc. The pioneers of the field such as Coulomb, Navier, Girad, Poisson, Stokes, St. Venant and others recognised that boundary conditions are constitutive equations that should be determined by the material on either side of the boundary.

The usual prescription of Dirichlet and Neumann conditions are often unsuitable for a realistic physical problem, for example the flow of polymers that stick-slip on the boundary. Recently non-standard boundary conditions have been considered from a rigorous mathematical perspective by Rao and Rajagopal.

The earliest work S.Asgar,M.Mudassar Gulzar, M.Ayub (2006) are derived an analytical study of the rotating flow of a third grade fluid past a porous plate with partial slip effects. It serves as a flow model for the study of polymers. The analytic solution has been determined using homotopy analysis method (HAM).

The work is extended to examine the effects of partial slip on the rotating flow past a uniformly porous plate. The fluid is incompressible and third grade. The numerical solution has been obtained using Finite difference method and finally focused on the effects of suction, blowing, third grade and slip parameter.

\section{A Study on rotating flow of a third grade fluid}

Consider the Cartesian coordinate system rotating uniformly with an angular velocity $\Omega$ about the zaxis , taken positive in the vertically upward direction and the plate coinciding with the plane $z=0$. The fluid flowing past a porous plate is third grade and incompressible. All the material parameters of the fluid are assumed to be constants .

For the rotating frame, the equation of momentum

$$
\rho\left[\frac{d V}{d t}+2 \Omega \times V+\Omega \times(\Omega \times V)\right]=\rho b+\operatorname{div} T
$$

is considered in which $\mathrm{T}$ is the Cauchy stress tensor for third grade fluid as given

$$
T=-p_{1} I+\mu A_{1}+\alpha_{1} A_{2}+\alpha_{2} A_{1}^{2}+\beta_{1} A_{3}+\beta_{2}\left(A_{1} A_{2}+A_{2} A_{1}\right)+\beta_{3}\left(t r A_{1}^{2}\right) A_{1}
$$

From the thermodynamical considerations, the material constants

$$
\mu \geq 0, \quad \alpha_{1} \geq 0, \quad\left|\alpha_{1}+\alpha_{2}\right| \leq \sqrt{24 \mu \beta_{3}}, \beta_{1}=0, \quad \beta_{2}=0, \quad \beta_{3} \geq 0
$$

must satisfied the equation(3.2).

under thermodynamical considerations, Cauchy stress tensor of a third grade fluid is

$$
T=-p_{1} I+\mu A_{1}+\alpha_{1} A_{2}+\alpha_{2} A_{1}^{2}+\beta_{3}\left(t r A_{1}^{2}\right) A_{1}
$$

For a uniform porous boundary, the continuity equation is satisfied if

$$
V=\left[u(z), v(z),-w_{0}\right]
$$

Where $\mathrm{u}$ and $\mathrm{v}$ are $\mathrm{x}$ - and $\mathrm{y}$ - components of velocity and $w_{0}>0\left(w_{0}<0\right)$ corresponding to suction (blowing ) velocity respectively .

In view of equation (4),(5) (1),(3) and equation of continuity

$$
\nabla \cdot V=0
$$

Then the equation (2) can be written as 


$$
\begin{array}{r}
\rho\left[-W_{0} \frac{d u}{d z}-2 v \Omega\right]=\mu \frac{d^{2} u}{d z^{2}}-\alpha_{1} W_{0} \frac{d^{3} u}{d z^{3}}+2 \beta_{3} \frac{d}{d z}\left[\frac{d u}{d z}\left\{\left(\frac{d u}{d z}\right)^{2}+\left(\frac{d v}{d z}\right)^{2}\right\}\right] \\
\rho\left[-W_{0} \frac{d v}{d z}+2 u \Omega\right]=2 \Omega U_{0} \rho+\mu \frac{d^{2} v}{d z^{2}}-\alpha_{1} W_{0} \frac{d^{3} u}{d z^{3}}+2 \beta_{3} \frac{d}{d z}\left[\frac{d v}{d z}\left\{\left(\frac{d u}{d z}\right)^{2}+\left(\frac{d v}{d z}\right)^{2}\right\}\right]
\end{array}
$$

Where $U_{0}$ denotes the uniform velocity outside the layer which is caused by the pressure gradient.

The approximate boundary conditions are

$$
\begin{gathered}
u=v=0 \text { at } z=0, u \rightarrow U_{0}, v \rightarrow 0 \text { as } z \rightarrow \infty \\
\text { Defining } \\
F=\frac{u+i v}{u_{0}}-1, F^{*}=\frac{u-i v}{u_{0}}-1
\end{gathered}
$$

equations (6) to (8) can be combined as

$$
2 i \Omega F-W_{0} \frac{d F}{d z}=\frac{1}{\rho}\left[\frac{d^{2} F}{d z^{2}}-\alpha W_{0} \frac{d^{3} F}{d z^{3}}+2 \beta_{3} \frac{d}{d z}\left\{\left(\frac{d F}{d z}\right)^{2} \frac{d F^{*}}{d z}\right\}\right]
$$

Subject to following boundary conditions

$$
F(z)=-1 \text { at } z=0, F(z) \rightarrow 0 \text { as } z \rightarrow \infty
$$

Where $F^{*}$ is conjugate of $\mathrm{F}$.

It is convenient to introduce the following dimensionless quantities

$$
\begin{gathered}
\hat{z}=\frac{\rho U_{0} Z}{\mu}, \widehat{F}=\frac{F}{U_{0}}, \widehat{W_{0}}=\frac{W_{0}}{U_{0}} \\
\widehat{\Omega}=\frac{\Omega \mu}{\rho U_{0}{ }^{2}}, \hat{\beta}=\beta_{3} \rho^{2} U_{0}{ }^{4}, \widehat{\alpha}=\frac{\alpha_{1} \rho U_{0}{ }^{2}}{\mu^{2}}
\end{gathered}
$$

After dropping hats, the resulting problem consists of conditions (11) and the following differential equation

$$
\frac{d^{2} F}{d z^{2}}-2 i \Omega F+W_{0}\left[\frac{d F}{d z}-\alpha \frac{d^{3} F}{d z^{3}}\right]+2 \beta \frac{d}{d z}\left[\left(\frac{d F}{d z}\right)^{2} \frac{d F^{*}}{d z}\right]=0
$$

Since equation (13) is a third -order differential equation which is higher than the governing equation of the Newtonian fluid. Therefore, introduce one more condition. The flow under consideration is in an unbounded domain, so by augmentation of the boundary conditions(11)

$$
\frac{d F}{d z} \rightarrow 0 \text { as } z \rightarrow \infty
$$

\section{Mathematical problem for the partial slip case}

Let an infinite porous plate at $\mathrm{z}=0$ bound a semi-infinite expanse $(\mathrm{Z}>0)$ of a third grade fluid which is assumed to be incompressible .Both the plate and the fluid does not rotate as solid body with constant angular velocity $\Omega$ about an axis normal to the plate .Consider Cartesian axes $\quad(\mathrm{x}, \mathrm{y}, \mathrm{z})$ such that the $\mathrm{z}$-axis is parallel to the common axes of rotating of the fluid . Since the plate is infinite in extent, all the physical quantities, expect the pressure, depend on $\mathrm{z}$ only for steady flow. Furthermore, the fluid adheres to the plate partially and thus motion of the fluid exhibits the slip condition.

The dimensionless governing problem is

$$
\frac{d^{2} F(z)}{d z^{2}}-2 i \Omega F(z)+w_{0}\left[\frac{d F(z)}{d z}-\alpha \frac{d^{3} F(z)}{d z^{3}}\right]=-2 \beta \frac{d}{d z}\left[\left(\frac{d F(z)}{d z}\right)^{2} \frac{d F^{*}(z)}{d z}\right] .
$$

The dimensionless governing partial slip case equation is

In equation (2)

$$
\begin{gathered}
\mathrm{F}(0)+1=\lambda_{1}\left[\frac{d F(z)}{d z}-\alpha w_{0} \frac{d^{2} F(z)}{d z^{2}}+2 \beta\left(\frac{d F(z)}{d z}\right)^{2} \frac{d F^{*}(z)}{d z}\right] \\
F(z)=0 \text { as } z \rightarrow \infty
\end{gathered}
$$

Is non-dimensional partial slip coefficient, $\tilde{\lambda}=\lambda \mu$ is slip length . In writing conditions (2), consider the following partial slip condition

$$
(u, v)-\left(U_{w}, V_{w}\right)=\lambda\left(T_{x z}, T_{y z}\right)
$$

\section{Rotating flows of a third grade fluid with partial slip}

The governing problem consist of

$$
\begin{gathered}
\frac{d^{2} F}{d z^{2}}-2 i \Omega F+w_{0}\left[\frac{d F}{d z}-\alpha \frac{d^{3} F}{d z^{3}}\right]+2 \beta \frac{d}{d z}\left[\left(\frac{d F}{d z}\right)^{2} \frac{d F^{*}}{d z}\right]=0 . \\
\mathrm{F}(0)+1=\lambda_{1}\left[\frac{d F(z)}{d z}-\alpha w_{0} \frac{d^{2} F(z)}{d z^{2}}+2 \beta\left(\frac{d F(z)}{d z}\right)^{2} \frac{d F^{*}(z)}{d z}\right] \\
F(z)=0 \text { as } z \rightarrow \infty
\end{gathered}
$$

Numerical solution by using finite difference equation

$$
\begin{aligned}
\left(F_{z}\right)_{i} & \simeq \frac{1}{2 h}\left[F_{i+1}-F_{i-1}\right] . \\
\left(F_{z z}\right)_{i} & \simeq \frac{1}{h^{2}}\left[F_{i+1}-2 F_{i}+F_{i-1}\right] . \\
\left(F_{z z z}\right)_{i} & \simeq \frac{1}{2 h^{3}}\left[F_{i+2}-2 F_{i+1}+2 F_{i-1}-F_{i-2}\right]
\end{aligned}
$$


The equation (20) reduced in the form

$$
\begin{aligned}
& \frac{1}{h^{2}}\left[F_{i+1}-2 F_{i}+F_{i-1}\right]-2 i \Omega F_{i}+w_{0}\left[\frac{1}{2 h}\left[F_{i+1}-F_{i-1}\right]-\frac{\alpha}{2 h^{3}}\left(F_{i+2}-2 F_{i+1}+2 F_{i-1}-F_{i-2}\right)\right]+ \\
& \frac{\beta}{2 h^{4}}\left[\begin{array}{c}
2\left(F_{i+1}-F_{i-1}\right)\left(F_{i+2}-2 F_{i+1}+F_{i}\right)\left(F_{i+1}{ }^{*}-F_{i-1}{ }^{*}\right)+ \\
\left(F_{i+1}-F_{i-1}\right)^{2}\left(F_{i+2}{ }^{*}-2 F_{i+1}{ }^{*}-F_{i}^{*}\right)
\end{array}\right]=0 \\
& \frac{1}{2 h^{3}}\left[\begin{array}{c}
2 h\left(F_{i+1}-2 F_{i}+F_{i-1}\right)-2 h^{3}\left(2 i \Omega F_{i}\right)+w_{0} h^{2}\left(F_{i+1}-F_{i-1}\right)-\alpha w_{0}\left(F_{i+2}-2 F_{i+1}+2 F_{i-1}-F_{i-2}\right)+ \\
\beta 2 h 42 F i+1-F i-1 F i+2-2 F i+1+F i F i+1 *-F i-1 * F i+1-F i-12 F i+2 *-2 F i+1 *-F i *=0 \\
\frac{1}{2 h^{3}}\left[\alpha w_{0}\left(F_{i-2}\right)+\left(2 h-w_{0} h^{2}-2 \alpha w_{0}\right)\left(F_{i-1}\right)-\left(4 h-4 h^{3} i \Omega\right) F_{i}+\left(2 h+w_{0} h^{2}+2 \alpha w_{0}\right) F_{i+1}-\right. \\
\alpha w 0 F i+2=0
\end{array}\right.
\end{aligned}
$$

Where $z_{i}-z_{i-1}$ is preferred to be 0.01 for the present calculations.

The iterative procedure applied to the non linear part of the above equation

$$
G_{i} F_{i-2}{ }^{(n+1)}+H_{i} F_{i-1}{ }^{(n+1)}+I_{i} F_{i}^{(n+1)}+J_{i} F_{i+1}{ }^{(n+1)}+K_{i} F_{i+2}{ }^{(n+1)}=L_{i}
$$

And the initial guess approximation is taken to be

$$
\begin{gathered}
F_{i}{ }^{(0)}=0,0 \leq i \leq Q . \\
G_{i}=\alpha w_{0} \\
H_{i}=2 h-w_{0} h^{2}-2 \alpha w_{0} \\
I_{i}=-4 h-4 h^{3} i \Omega \\
J_{i}=2 h+w_{0} h^{2}+2 \alpha w_{0} \\
K_{i}=-\alpha w_{0} \\
L_{i}=-\frac{\beta}{h}\left[\begin{array}{c}
2\left(F_{i+1}{ }^{(n)}-F_{i-1}{ }^{(n)}\right)\left(F_{i+2}{ }^{(n)}-2 F_{i+1}{ }^{(n)}+F_{i}{ }^{(n)}\right)\left(F_{i+1}{ }^{*(n)}-F_{i-1}{ }^{*(n)}\right) \\
+\left(F_{i+1}{ }^{(n)}-F_{i-1}{ }^{(n)}\right)^{2}\left(F_{i+2}{ }^{(n)}-2 F_{i+1}{ }^{(n)}+F_{i}{ }^{(n)}\right)
\end{array}\right]
\end{gathered}
$$

For $i=1$,equation (28) is

$$
G_{i} F_{-1}{ }^{(n+1)}+H_{1}{F_{0}}^{(n+1)}+I_{1}{F_{1}}^{(n+1)}+J_{1}{F_{2}}^{(n+1)}+K_{1} F_{3}{ }^{(n+1)}=L_{1}
$$

The value of $\mathrm{F}$ at the fictitious point $Z_{-1}$ is approximated by means of the langrange polynomial of third degree

Where

$$
\begin{aligned}
F_{-1}{ }^{(n+1)} & =X_{0} F_{0}{ }^{(n+1)}+X_{1} F_{1}{ }^{(n+1)}+X_{2} F_{2}{ }^{(n+1)}+X_{3} F_{3}{ }^{(n+1)} \\
X_{0} & =\left(\frac{Z_{-1}-Z_{1}}{Z_{0}-Z_{1}}\right)\left(\frac{Z_{-1}-Z_{3}}{Z_{0}-Z_{3}}\right) \\
X_{1} & =\left(\frac{Z_{-1}-Z_{0}}{Z_{1}-Z_{0}}\right)\left(\frac{Z_{-1}-Z_{2}}{Z_{1}-Z_{2}}\right)\left(\frac{Z_{-1}-Z_{3}}{Z_{1}-Z_{3}}\right) \\
X_{2} & =\left(\frac{Z_{-1}-Z_{0}}{Z_{2}-Z_{0}}\right)\left(\frac{Z_{-1}-Z_{1}}{Z_{2}-Z_{1}}\right)\left(\frac{Z_{-1}-Z_{3}}{Z_{2}-Z_{3}}\right) \\
X_{3} & =\left(\frac{Z_{-1}-Z_{0}}{Z_{3}-Z_{0}}\right)\left(\frac{Z_{-1}-Z_{1}}{Z_{3}-Z_{1}}\right)\left(\frac{Z_{-1}-Z_{2}}{Z_{3}-Z_{2}}\right)
\end{aligned}
$$

Substitution of equation (37) into (36) yields

$$
\begin{aligned}
& G_{i}\left(X_{0} F_{0}{ }^{(n+1)}+X_{1} F_{1}^{(n+1)}+X_{2} F_{2}^{(n+1)}+X_{3} F_{3}^{(n+1)}\right)+H_{1} F_{0}{ }^{(n+1)}+I_{1} F_{1}^{(n+1)}+J_{1} F_{2}^{(n+1)}+K_{1} F_{3}^{(n+1)}=L_{1} \\
& \left(G_{1} X_{0}+H_{1}\right) F_{0}^{(n+1)}+\left(G_{1} X_{1}+I_{1}\right) F_{1}^{(n+1)}+\left(G_{1} X_{2}+J_{1}\right) F_{2}^{(n+1)}+\left(G_{1} X_{3}+K_{1}\right) F_{3}^{(n+1)}=L_{1}
\end{aligned}
$$

Now $F_{0}^{(n+1)}=F_{0}$ is know as fixed, so the equation (43) can be written as

In which

$$
I_{1}^{\prime} F_{1}^{(n+1)}+J_{1}^{\prime} F_{2}^{(n+1)}+K_{1}^{\prime} F_{3}^{(n+1)}=L_{1}^{\prime}
$$

For $i=2$ in equation (28)

$$
\begin{gathered}
I_{1}^{\prime}=G_{1} X_{1}+I_{1} \\
J_{1}^{\prime}=G_{1} X_{2}+J_{1} \\
K_{1}^{\prime}=G_{1} X_{3}+K_{1} \\
L_{1}^{\prime}=L_{1}-G_{1} X_{0}+H_{1}
\end{gathered}
$$

$$
G_{2} F_{0}^{(n+1)}+H_{2} F_{1}^{(n+1)}+I_{2} F_{2}^{(n+1)}+J_{2} F_{3}^{(n+1)}+K_{2} F_{4}^{(n+1)}=L_{2}
$$

Since $F_{0}^{(n+1)}=F_{0}$ is known thus from the above equation (49) can be written as

Where

$$
\begin{gathered}
H_{2} F_{1}^{(n+1)}+I_{2} F_{2}^{(n+1)}+J_{2} F_{3}^{(n+1)}+K_{2} F_{4}^{(n+1)}=L_{2}^{\prime} \\
L_{2}^{\prime}=L_{2}-G_{2} F_{0}
\end{gathered}
$$

For $3 \leq i \leq Q-3$, the equations are

For $i=Q-2$, the equation(52) becomes

$$
G_{i} F_{i-2}^{(n+1)}+H_{i} F_{i-1}^{(n+1)}+I_{i} F_{i}^{(n+1)}+J_{i} F_{i+1}^{(n+1)}+K_{i} F_{i+2}^{(n+1)}=L_{i}
$$




$$
G_{Q-2} F_{Q-4}^{(n+1)}+H_{Q-2} F_{Q-3}^{(n+1)}+I_{Q-2} F_{Q-2}^{(n+1)}+J_{Q-2} F_{Q-1}^{(n+1)}+K_{Q-2} F_{Q}^{(n+1)}=L_{Q-2}
$$

Since $F_{Q}^{(n+1)}=F_{Q}$ is known, so the equation (53) is

When

$$
\begin{gathered}
G_{Q-2} F_{Q-4}^{(n+1)}+H_{Q-2} F_{Q-3}^{(n+1)}+I_{Q-2} F_{Q-2}^{(n+1)}+J_{Q-2} F_{Q-1}^{(n+1)}=L_{Q-2}^{\prime} \\
L_{Q-2}^{\prime}=L_{Q-2}-K_{Q-2} F_{Q}
\end{gathered}
$$

For $=Q-1$, the equation (52) becomes

$$
G_{Q-1} F_{Q-3}^{(n+1)}+H_{Q-1} F_{Q-2}^{(n+1)}+I_{Q-1} F_{Q-1}^{(n+1)}+J_{Q-1} F_{Q}^{(n+1)}+K_{Q-1} F_{Q+1}^{(n+1)}=L_{Q-1}
$$

To find the value of $L_{Q-1}$, we must have the value of $F_{Q+1}$. Now augmentation of the boundary condition

$$
\frac{\partial F}{\partial Z}=0 \quad \text { as } \quad Z \rightarrow \infty
$$

Yields a well posed problem. The boundary condition is discretized to give

Thus for $=Q-1$, the equation (56) becomes

$$
F_{Q+1}=F_{Q} \text { i.e. } F_{Q+1}^{(n+1)}=F_{Q}^{(n+1)}
$$

Where

$$
\begin{gathered}
G_{Q-1} F_{Q-3}^{(n+1)}+H_{Q-1} F_{Q-2}^{(n+1)}+I_{Q-1} F_{Q-1}^{(n+1)}=L_{Q-1}^{\prime} \\
L_{Q-1}^{\prime}=L_{Q-1}-\left(J_{Q-1}+K_{Q-1}\right) F_{Q}
\end{gathered}
$$

It is noted that there are $Q-1$ equations in $Q-1$ unknowns and in matrix form

$$
\left[\begin{array}{ccccccccccc}
I_{1}^{\prime} & J_{1}^{\prime} & K_{1}^{\prime} & 0 & 0 & 0 & 0 & 0 & . & \cdot & 0 \\
H_{2} & I_{2} & J_{2} K_{2} & 0 & 0 & 0 & 0 & \cdot & \cdot & 0 \\
G_{3} & H_{3} & I_{3} J_{3} & K_{3} & 0 & 0 & 0 & \cdot & \cdot & 0 \\
\cdot & \cdot & \cdot & \cdot & \cdot & \cdot & \cdot & \cdot & \cdot & \cdot \\
0 & \cdot & 0 & G_{i} & H_{i} & I_{i} & J_{i} & K_{i} & 0 & \cdot & 0 \\
\cdot & \cdot & \cdot & \cdot & \cdot & \cdot & \cdot & \cdot & \cdot & \cdot & \cdot \\
0 & 0 & 0 & \cdot & \cdot & 0 & G_{Q-3} & H_{Q-3} & I_{Q-3} & J_{Q-3} & K_{Q-3} \\
0 & 0 & 0 & 0 & \cdot & \cdot & 0 & G_{Q-2} & H_{Q-2} & I_{Q-2} & J_{Q-2} \\
0 & 0 & 0 & \cdot & . & 0 & G_{Q-1} H_{Q-1} & I_{Q-1}
\end{array}\right]\left[\begin{array}{c}
F_{1}^{(n+1)} \\
F_{2}^{(n+1)} \\
F_{3}^{(n+1)} \\
\cdot \\
F_{i}^{(n+1)} \\
\cdot \\
F_{Q-3}^{(n+1)} \\
F_{Q-2}^{(n+1)} \\
F_{Q-1}^{(n+1)}
\end{array}\right]=\left[\begin{array}{c}
L_{1}^{\prime} \\
L_{2} \\
L_{3} \\
\cdot \\
L_{i} \\
\cdot \\
L_{Q-2} \\
L_{Q-2}^{\prime} \\
L_{Q-1}^{\prime}
\end{array}\right]
$$

Matrix involved in above equation is pentadiagonal.

It is observed that the partial slip boundary condition (21) is also highly nonlinear. Thus following the same procedure as adopted for discretization of equation (20), equation (21) may be discretized as

When

$$
\begin{aligned}
F_{0}^{(n)}= & r_{1} F_{1}{ }^{(n)}+r_{2} F_{2}{ }^{(n)}+r_{3}\left[-1+E_{1} T^{n}\right] \\
& r_{0}=h^{2}+\lambda\left(h+\alpha W_{0}\right) \\
& r_{1}=\lambda\left(h+2 \alpha W_{0}\right) / r_{0} \\
& r_{2}=-\alpha W_{0} \lambda / r_{0} \\
r_{3} & =h^{2} / r_{0} \\
E_{1} T^{(n)}= & \frac{2 \beta}{h^{3}} \lambda\left[\left(F_{1}{ }^{(n)}-F_{0}{ }^{(n)}\right)^{2}\left(F_{1}{ }^{*(n)}-F_{0}{ }^{*(n)}\right)\right]
\end{aligned}
$$

To evaluate $F_{0}^{(n+1)}$, first take $E_{1} T^{(n+1)}=E_{1} T^{(n)}$ in the system of algebraic equations and the solution of the system is sought for the unknown values of $F_{i}^{(n+1)}, \mathrm{i}=1,2,3 \ldots \ldots, Q-1$. Then update $F_{0}^{(n+1)}$ by using iterative method as follows

$$
\begin{aligned}
& F_{0,(k+1)}^{(n+1)}=r_{1} F_{1}{ }^{(n+1)}+r_{2} F_{2}{ }^{(n+1)}+r_{3}\left[\frac{2 \beta}{h^{3}} \lambda\left[\left(F_{1}^{\left({ }^{(n+1)}\right.}-F_{0,(k)}{ }^{(n+1)}\right)^{2}\left(F_{1}{ }^{(n+1)}-F_{0,(k)}{ }^{*(n+1)}\right)-1\right]\right] \\
& \text { Where } \\
& F_{0,(1)}^{(n+1)}=F_{0}^{(n+1)}
\end{aligned}
$$

This iterative procedure is continued until $F_{0,(k+1)}^{(n+1)} \approx F_{0,(k)}^{(n+1)}$

For $=1, \quad i=2,3 \leq i \leq Q-3, i=Q-2$, and $i=Q-1$,

Equation becomes

$$
\begin{gathered}
I_{1}^{\prime} F_{1}{ }^{(n+1)}+J_{1}^{\prime} F_{2}{ }^{(n+1)}+K_{1}^{\prime} F_{3}{ }^{(n+1)}=L_{1}^{\prime} \\
H_{2}^{\prime} F_{1}{ }^{(n+1)}+I_{1}^{\prime} F_{2}{ }^{(n+1)}+J_{2} F_{3}{ }^{(n+1)}+K_{2} F_{4}{ }^{(n+1)}=L_{2}^{\prime} \\
G_{i} F_{i-2}{ }^{(n+1)}+H_{i} F_{i-1}{ }^{(n+1)}+I_{i} F_{i}{ }^{(n+1)}+J_{i} F_{i+1}\left({ }^{(n+1)}+K_{i} F_{i+2}{ }^{(n+1)}=L_{i}\right. \\
G_{Q-2} F_{Q-4}{ }^{(n+1)}+H_{Q-2} F_{Q-3}{ }^{(n+1)}+I_{Q-2} F_{Q-2}{ }^{(n+1)}+J_{Q-2} F_{Q-1}{ }^{(n+1)}=L_{Q-2}^{\prime} \\
G_{Q-1} F_{Q-3}{ }^{(n+1)}+H_{Q-1} F_{Q-2}{ }^{(n+1)}+I_{Q-1} F_{Q-1}{ }^{(n+1)}=L_{Q-1}^{\prime}
\end{gathered}
$$

In which $G_{i}, H_{i}, I_{i}, J_{i}, K_{i}$ and $L_{i}$ are given through equations ( 30) to (34)

In the above equations 


$$
\begin{aligned}
& I_{1}^{\prime}=G_{1} X_{1}+I_{1}+r_{1}\left(H_{1}+X_{0} G_{1}\right) \\
& J_{1}^{\prime}=G_{1} X_{2}+J_{1}+r_{2}\left(H_{1}+X_{0} G_{1}\right) \\
& K_{1}^{\prime}=G_{1} X_{3}+K_{1} \\
& L_{1}^{\prime}=L_{1}-\left(G_{1} X_{0}+H_{1}\right)\left[r_{3}\left(E_{1} T^{(n)}-1\right)\right] \\
& H_{2}^{\prime}=H_{2}+r_{1} G_{2} \\
& I_{2}^{\prime}=I_{2}+r_{2} G_{2} \\
& L_{2}^{\prime}=L_{2}-G_{2}\left[r_{3}\left(E_{1} T^{(n)}-1\right)\right] \\
& L_{Q-2}^{\prime}=L_{Q-2}-K_{Q-2} F_{Q} \\
& L_{Q-1}^{\prime}=L_{Q-1}-\left(L_{Q-1}+K_{Q-2}\right) F_{Q} \\
& {\left[\begin{array}{ccccccccccc}
I_{1}^{\prime} & J_{1}^{\prime} & K_{1}^{\prime} & 0 & 0 & 0 & 0 & 0 & \cdot & \cdot & 0 \\
H_{2}{ }^{\prime} & I_{2} & J_{2} & K_{2} & 0 & 0 & 0 & 0 & \cdot & \cdot & 0 \\
\cdot & \cdot & \cdot & \cdot & \cdot & \cdot & \cdot & \cdot & \cdot & \cdot & 0 \\
\cdot & \cdot & \cdot & \cdot & \cdot & \cdot & \cdot & \cdot & \cdot & \cdot & \cdot \\
0 & \cdot & 0 & G_{i} & H_{i} & I_{i} & J_{i} & K_{i} & 0 & \cdot & 0 \\
\cdot & \cdot & \cdot & \cdot & \cdot & \cdot & \cdot & \cdot & \cdot & \cdot & \cdot \\
0 & 0 & 0 & \cdot & \cdot & \cdot & \cdot & \cdot & \cdot & \cdot & \cdot \\
0 & 0 & 0 & 0 & \cdot & \cdot & 0 & G_{Q-2} & H_{Q-2} & I_{Q-2} & J_{Q-2} \\
0 & 0 & 0 & 0 & 0 & \cdot & \cdot & 0 & G_{Q-1} & H_{Q-1} & I_{Q-1}
\end{array}\right]\left[\begin{array}{c}
F_{1}^{(n+1)} \\
F_{2}^{(n+1)} \\
\cdot \\
\cdot \\
F_{i}^{(n+1)} \\
\cdot \\
\cdot \\
F_{Q-2}^{(n+1)} \\
F_{Q-1}^{(n+1)}
\end{array}\right]=\left[\begin{array}{c}
L_{1}^{\prime} \\
L_{2}^{\prime} \\
\cdot \\
\cdot \\
L_{i} \\
\cdot \\
\cdot \\
L_{Q-2}^{\prime} \\
L_{Q-1}^{\prime}
\end{array}\right]}
\end{aligned}
$$

The matrix form of the above set of $Q-1$ equations in $Q-1$ unknowns is a pentadiagonal.

\section{Result and discussion}

The variation of velocity components for various values of partial slip parameter $\lambda_{1}$ for the third grade fluid are discussed. The effect of slip parameter in the third grade fluid are shown by graphs (1) to (4).

\section{Graphs}

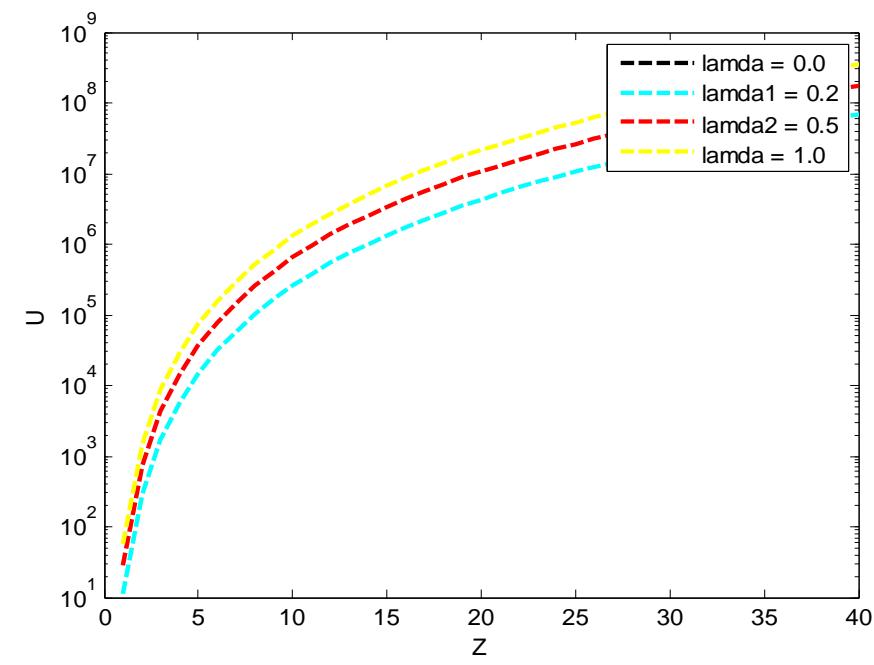

Fig. (1) $\alpha=\beta=1, \Omega=w_{0}=0.5$

The variation of velocity components for various values of partial slip parameter $\lambda_{1}$ for viscous fluid with fixed $\alpha, \beta, \Omega$ and $w_{0}$. 


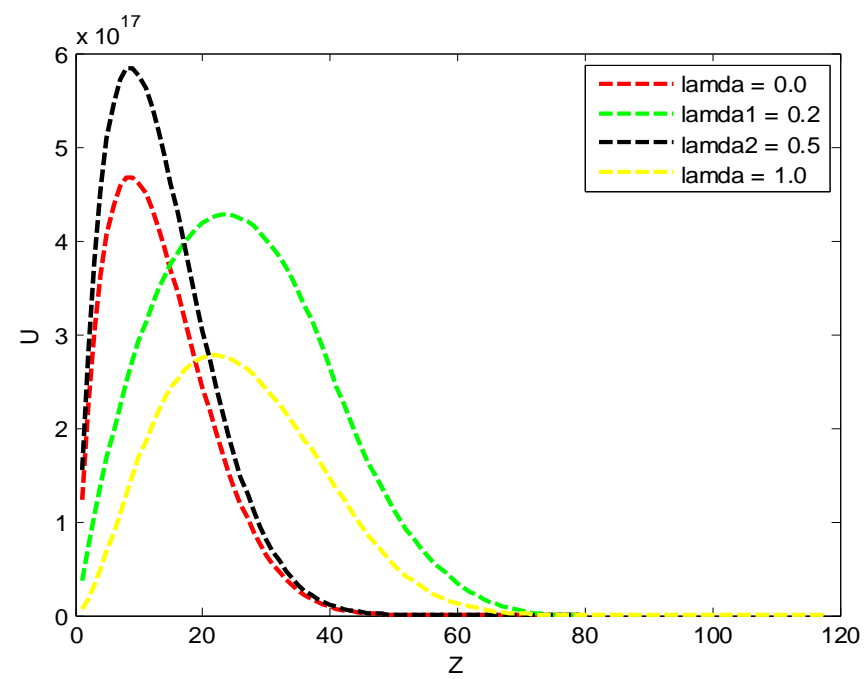

Fig. (2) $\alpha=\beta=1, \Omega=w_{0}=0.5$

The variation of velocity components for various values of partial slip parameter $\lambda_{1}$ for viscous fluid with fixed $\alpha, \beta, \Omega$ and $w_{0}$.

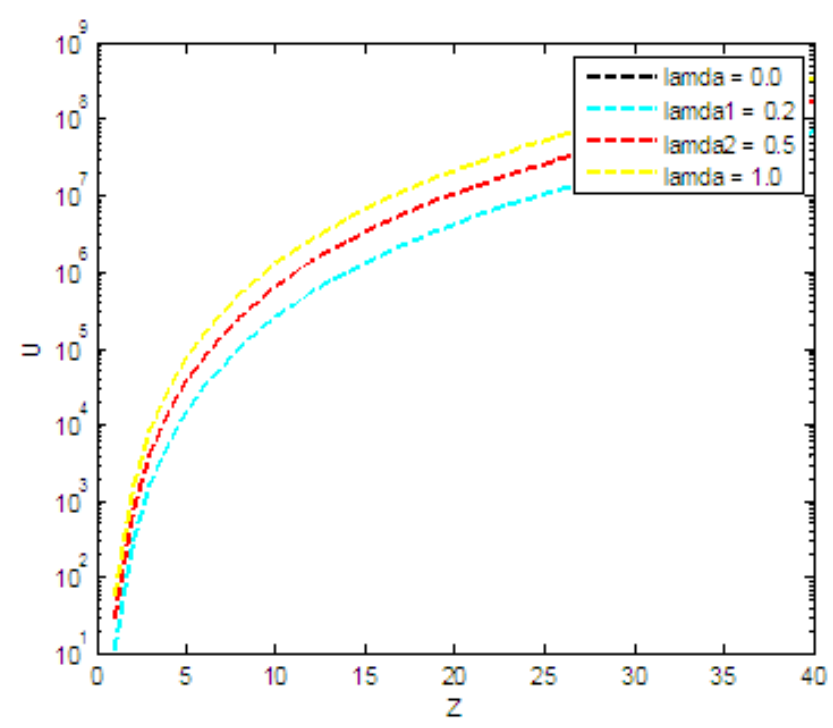

Fig. (3) $\alpha=\beta=0, \Omega=w_{0}=0.5$

The variation of velocity components for various values of partial slip parameter $\lambda_{1}$ for viscous fluid with fixed $\alpha, \beta, \Omega$ and $w_{0}$.

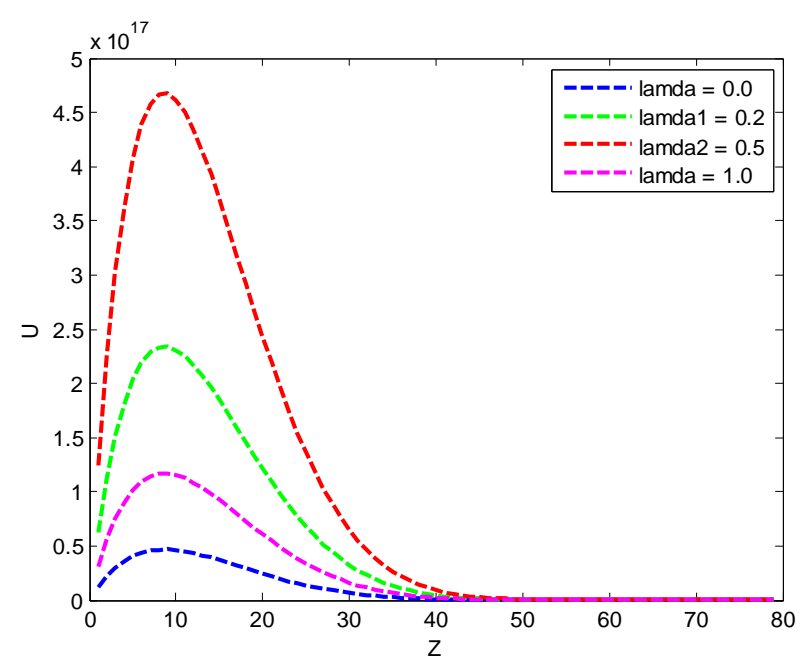

Fig. (4) $\alpha=\beta=0, \Omega=w_{0}=0.5$ 
The variation of velocity components for various values of partial slip parameter $\lambda_{1}$ for viscous fluid with fixed $\alpha, \beta, \Omega$ and $w_{0}$.

\section{Conclusion}

It is found that the velocity components $u$ and $v$ increases with an increase in slip parameter.

\section{References}

[1]. Asghar.S, Mudassar Gulzar.M ,Hayat.T,Rotating flow of a third grade fluid by homotopy analysis method ,Appl.Math.and Comput.,165,213-221(2005)

[2]. Asgar .S, Mudassar Gulzar .M and Ayub.M, Effects of partial slip on flow of a third grade fluid,Acta Mech.Sinica.,22,195198(2006).

[3]. Rao.I.J and Rajagopal.K.R, The effect of the slip boundary condition on the flow of fluids in a channel, Acta Mech.,135,113126(1999).

[4]. Hayat.T, Nadeem.S, Asghar.S and Siddiqui.A.M, Fluctuating flow of a third grade fluid on a porous plate ,Int.J.Non -Linear Mech.,368,901-906 (2001).

[5]. Hayat.T, Nadeem.S, Asghar.S and Siddiqui.A.M,MHD rotating flow of a third grade fluid on an oscillating plate,Acta Mech.,152,177-190 (2001).

[6]. Ayub.M,Rasheed .A and Hayat.T, Exact flow of a third grade fluid past a porous plate using homotopy analysis method ,Int.J.Engng.Sci.,41,2091-2103 (2003).

[7]. Smith.G.D,Numerical Solutions of Partial Differential Equations:Finite Difference methods.Oxford University Press,New York(1985). 